\section{Studying cooling curves with a smartphone}

Manuela Ramos Silva, CFisUC, Department of Physics, FCTUC, Universidade de Coimbra, P-3004-516 Coimbra, Portugal; manuela@ uc.pt, Pablo Martín-Ramos, CFisUC, Department of Physics, FCTUC, Universidade de Coimbra, P-3004-516 Coimbra, Portugal; EPS, Universidad de Zaragoza, Ctra. Cuarte s/n, 22071 Huesca, Spain; pmr@unizar.es, Pedro Pereira da Silva, CFisUC, Department of Physics, FCTUC, Universidade de Coimbra, P-3004-516 Coimbra, Portugal; psidonio@uc.pt

$\mathrm{T}$ his paper describes a simple procedure for the study of the cooling of a spherical body using a standard thermometer and a smartphone. Experiments making use of smartphone sensors have been described before, contributing to an improved teaching of classical mechanics, ${ }^{1-11}$ but rarely expand to thermodynamics. ${ }^{12-14}$ In this experiment, instead of using a smartphone camera to slow down a fast movement, we are using the device to speed up a slow process. For that we propose the use of the free app Framelapse ${ }^{15}$ to take periodic pictures (in the form of a time-lapse video) and then the free app VidAnalysis ${ }^{16}$ to track the position of the mercury inside the thermometer, thus effortlessly tracking the temperature of a cooling body (Fig. 1).

The experiment consists of filling a round-bottom flask

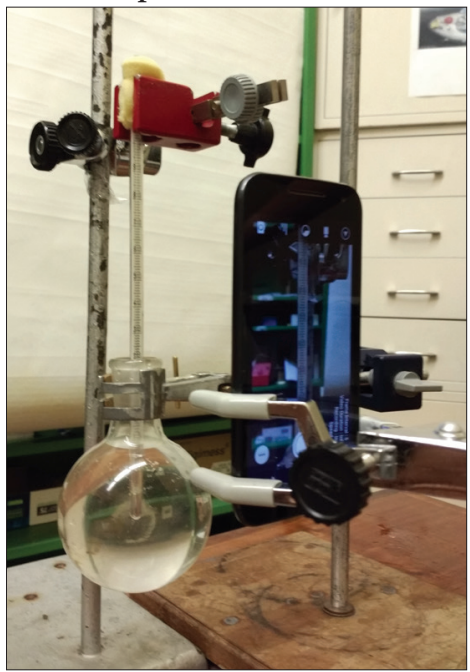

Fig. 1. Photograph of the experimental setup. (five flasks with standard sizes-50, 100, 250, 500, and $1000 \mathrm{~mL}$ - were borrowed from the chemistry lab) with hot water, placing a mercury thermometer in the opening and taking periodic pictures of it. The Framelapse app allows the user to set the time interval between pictures (30 s was found to be a suitable choice) and it is a way of automatically monitoring a lengthy experiment (ca. two hours for the 1-L flask).

The video is then processed in the smartphone using VidAnalysis. This intuitive and easy-to-use app requires the setting of the axes, a length scale-which can be done by using the thermometer scale-and the tracking of the mercury position through screen touching (Fig. 2, right), frame by frame, generating temperature-vs.-time graphs. The data can then be exported into a CSV file. The file can be further manipulated to allow logarithmization or fitting of the experimental curves directly

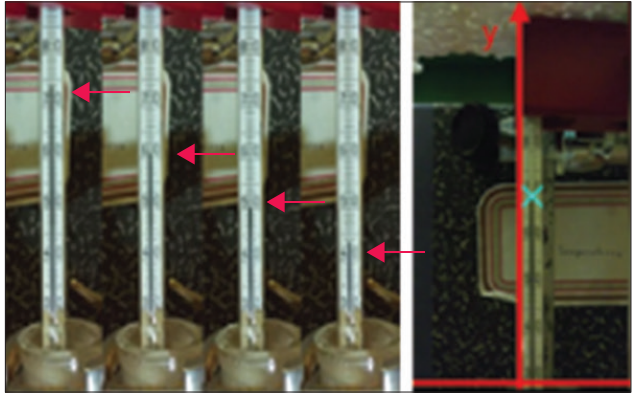

Fig. 2. Some of the frames taken during the cooling of the hot water with Framelapse app (left) and mercury position tracking with VidAnalysis app (right).

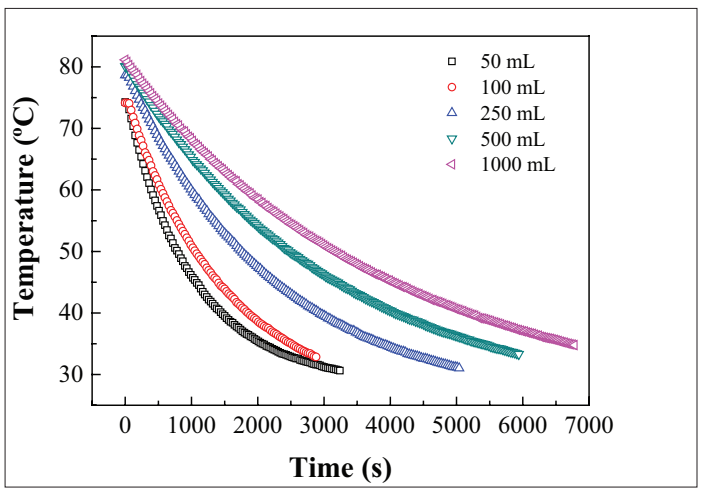

Fig. 3. Plot of decay of water temperature as a function of time for the five round-bottom flasks.

in the smartphone using the Google Sheets app (other popular free apps cannot open CSV files) or in a computer with OpenOffice Calc or MS Excel.

\section{Newton's law of cooling}

The temperature of a hot object placed in a cooler surrounding will slowly decrease until it matches that of the environment. It decreases by a combination of three phenomena: conduction, convection, and radiation. The heat flow coming from conduction and convection depends linearly on the difference of the temperature of the object and that of its surroundings: ${ }^{17}$

$$
\frac{d Q}{d t} \propto\left(T_{\text {object }}-T_{\text {env }}\right) .
$$

On the other hand, the heat flow coming from radiation is ruled by the Stefan-Boltzmann law, with a dependence on the fourth power of $T$. The net heat flow from the object radiation and the surrounding radiation is linearly dependent on $\Delta T$ only for very small temperature differences.

Anyhow, for cases where the radiative processes are not predominant, as the one discussed herein, Newton's cooling law can be used to model the system:

$$
T_{\text {object }}(t)=T_{\text {env }}+\left(T_{\text {object }}(0)-T_{\text {env }}\right) \cdot \exp (-t / \tau)
$$


with

$$
\tau=M c / h S \text {, }
$$

$M$ being the mass of the body, $S$ the surface area, $c$ the specific heat capacity per unit mass, and $h$ the convective heat transfer parameter.

Figure 3 shows the variation of the temperature of our flasks filled with water; a least-squares fit using a simple exponential (LOGEST function in Google Sheets app) yields $T_{\text {env }}=25.21(5)^{\circ} \mathrm{C}, T_{\text {object }}(0)-T_{\text {env }}=55.72(4){ }^{\circ} \mathrm{C}$ and $3877(8) \mathrm{s}$ for the decay time constant $\tau$.

\section{Linear dependence on $M / S$ quotient}

By applying logarithms on both sides of Eq. (2), a linear dependence is obtained:

$$
\ln \left(T_{\text {object }}-T_{\text {env }}\right)=\left(T_{\text {object }}(0)-T_{\text {env }}\right)-t / \tau \text {. }
$$

A fit of the plotted data (using the LINEST function) yields the slope value, that is, the reciprocal of $\tau$. On its own, $\tau$ depends on four parameters [Eq. (3)]: $c$ is kept constant and $h$ is also approximately constant (since the conditions of the experiment were kept as close to each other as possible: a fan at medium speed was kept in a corner of the room to keep the ambient air conditions as similar as possible in all the runs). ${ }^{18}$ The other two parameters, $M$ and $S$, were changing in the five performed runs. Their quotient depends only on the radius of the outer surface:

$$
\frac{M}{S}=\frac{\rho \frac{4}{3} \pi R^{3}}{4 \pi R^{2}}=\frac{\rho}{3} R,
$$

where $\rho$ is the density of water and $R$ is the radius.

The $\tau$ values obtained using this procedure are summarized in Table I and plotted in Fig. 4.

Table I. Mass, outer surface, and decay constant values for the five runs.

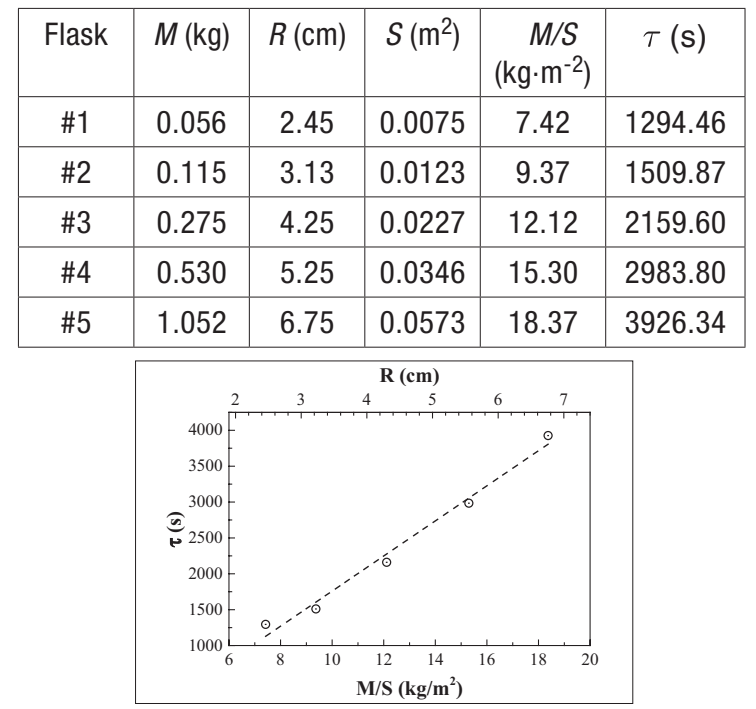

Fig. 4. Plot of $\tau$ as a function of $M / S$ quotient (or $R$, for simplicity we have just used the radius of the flask) with a least-squares linear fit.
The slope of the $\tau$ vs. $M / S$ linear fit (with $R^{2}=0.9853$ ) yields the constant $c / h=244.6 \mathrm{~m}^{2} \mathrm{~s} / \mathrm{kg}$, or using $c=4.186$ $\mathrm{J} /\left(\mathrm{g}{ }^{\circ} \mathrm{C}\right)$, one gets $h \sim 58 \mathrm{~W} /\left(\mathrm{m}^{2}{ }^{\circ} \mathrm{C}\right)$, which is in the expected range according to Ref. 17. $h$ is dependent on the geometry, flow orientation, surface roughness, and combination of material/fluid.

The proposed experiment can be useful for the introduction of exponential functions at introductory levels, and can easily be changed to investigate the heat capacity of materials by letting blocks of different materials cool in air or water. ${ }^{19-21}$ Since the procedure can readily be adapted to allow the simultaneous tracking of several thermometers, it may also prove useful for investigating the cooling of a nonhomogeneous body (e.g., simulating the estimation of the time since death of a human body, ${ }^{22}$ if our students are CSI fans!). This experiment benefits from the small size and portability of the setup, from running autonomously once set (freeing both students and teachers to other tasks) and from using a device that students bring voluntarily to class (although a sophisticated camera plus a computer can replace the smartphone). Profiting from the above characteristics, more complex experiments in non-ambient conditions (e.g., inside a fridge) can also be envisaged.

\section{Acknowledgments}

CFisUC gratefully acknowledges funding from FCT Portugal through Grant UID/FIS/04564/2016. P.M-R would like to thank Santander Universidades for its financial support through the Becas Iberoamérica Jóvenes Profesores e Investigadores, España 2017 scholarship program.

\section{References}

1. J. Chevrier, L. Madani, S. Ledenmat, and A. Bsiesy, “Teaching classical mechanics using smartphones," Phys. Teach. 51, 376 (Sept. 2013).

2. J. Hall, “More smartphone acceleration," Phys. Teach. 51, 6 (Jan. 2013).

3. M. Monteiro, C. Stari, C. Cabeza, and A. C. Marti, “The Atwood machine revisited using smartphones," Phys. Teach. 53, 373-374 (Sept. 2015).

4. A. Shakur and J. Kraft, "Measurement of Coriolis acceleration with a smartphone," Phys. Teach. 54, 288-290 (May 2016).

5. P. Vogt and J. Kuhn, "Analyzing simple pendulum phenomena with a smartphone acceleration sensor," Phys. Teach. 50, 439 (Oct. 2012).

6. P. Vogt, J. Kuhn, and S. Müller, "Experiments using cell phones in physics classroom education: The computer-aided $g$ determination," Phys. Teach. 49, 383-384 (Sept. 2011).

7. S. Becker, P. Klein, and J. Kuhn, "Video analysis on tablet computers to investigate effects of air resistance," Phys. Teach. 54, 440-441 (Oct. 2016).

8. S. Gröber, P. Klein, and J. Kuhn, "Video-based problems in introductory mechanics physics courses," Eur. J. Phys. 35 (5), 055019 (2014).

9. P. Klein, J. Kuhn, A. Müller, and S. Gröber, "Video Analysis Exercises in Regular Introductory Physics Courses: Effects of Conventional Methods and Possibilities of Mobile Devices," in 
Multidisciplinary Research on Teaching and Learning, edited by Wolfgang Schnotz, Alexander Kauertz, Heidrun Ludwig, Andreas Müller, and Johanna Pretsch (Palgrave Macmillan UK, London, 2015), pp. 270-288.

10. P. Klein, S. Gröber, J. Kuhn, and A. Müller, "Video analysis of projectile motion using tablet computers as experimental tools," Phys. Educ. 49 (1), 37-40 (2014).

11. V. Pereira, P. Martín-Ramos, P. P. da Silva, and M. R. Silva, "Studying 3D collisions with smartphones," Phys. Teach. 55, 312-313 (May 2017).

12. D. Brown and A. J. Cox, "Innovative uses of video analysis," Phys. Teach. 47 (3), 145-150 (March 2009).

13. L. Moggio, P. Onorato, L. M. Gratton, and S. Oss, "Time-lapse and slow-motion tracking of temperature changes: Response time of a thermometer," Phys. Educ. 52 (2), 023005 (2017).

14. M. P. Strzys, S. Kapp, M. Thees, J. Kuhn, P. Lukowicz, P. Knierim, and A. Schmidt, "Augmenting the thermal flux experiment: A mixed reality approach with the HoloLens," Phys. Teach. 55, 376-377 (Sept. 2017).

15. Neximo Labs, Framelapse-Time Lapse Camera (Google Play, 2017), https://play.google.com/store/apps/details?id=com.Nishant.Singh.DroidTimelapse (temporary web address).
16. tsaedek, VidAnalysis free v. 1.63 (Google Play, 2017), https:// play.google.com/store/apps/details?id=com.vidanalysis.free (temporary web address).

17. T. L. Bergman and A. Lavine, Fundamentals of Heat and Mass Transfer, 8th ed. (Wiley, Inc., Hoboken, NJ, 2017).

18. This is a simplified model for the actual pathways for the transfer of energy. In reality, three major pathways come into play: the free convection from the warm water to the glass flask, the conduction through the flask walls, and the forced convection from the flask to the room air. For advanced classes, all 3 phenomena can be taken in consideration and their relative effect pondered by changing the fan speed.

19. C. R. Mattos and A. Gaspar, "Introducing specific heat through cooling curves," Phys. Teach. 40, 415-416 (Oct. 2002).

20. G. Planinšič and M. Vollmer, "The surface-to-volume ratio in thermal physics: From cheese cube physics to animal metabolism," Eur. J. Phys. 29 (2), 369-384 (2008).

21. J. B. Will, N. P. Kruyt, and C. H. Venner, "An experimental study of forced convective heat transfer from smooth, solid spheres," Int. J. Heat Mass Transfer 109, 1059-1067 (2017).

22. C. Leinbach, "Beyond Newton's law of cooling - Estimation of time since death," Int. J. Math. Educ. Sci. Technol. 42 (6), 765-774 (2011).

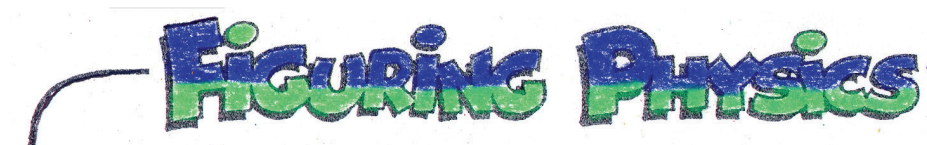

While driving along the highway you experience a head-on collision with a fat lovebug that splatters onto your windshield-SPLAT! Which of the following is exerted on both the bug and the car windshield with equal magnitudes?
A. Force of impact.
B. Impuise.
C. Change in momentum.
D. All of these.
E. None of these.

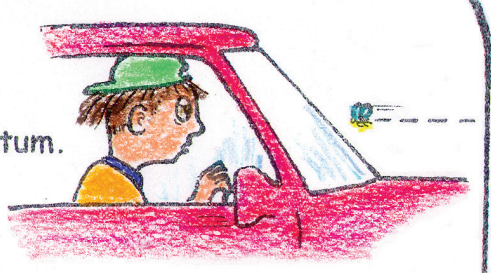

\section{Answer: D}

In accord with Newton's third law, the force that the bug exerts against the windshield is equal and opposite to the force that the windshield unfortunately exerts on the bug. Since the duration of the splat is the same for each, the impulse has the same magnitude for both bug and car. In accord with the impulse-momentum relationship. Ft $=\Delta m v$, the change in momentum is the same for both.

What is different is the accelerations for each! You may not notice the reduced speed of your car, but it's there. In contrast, the abrupt change in flying speed of the bug is very noticeable-actually lethal!

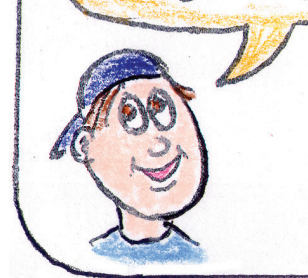

Can you see the wonder of physics here? Although so much is different for the bug and your car, the amount of force on each is the same. Amazing!
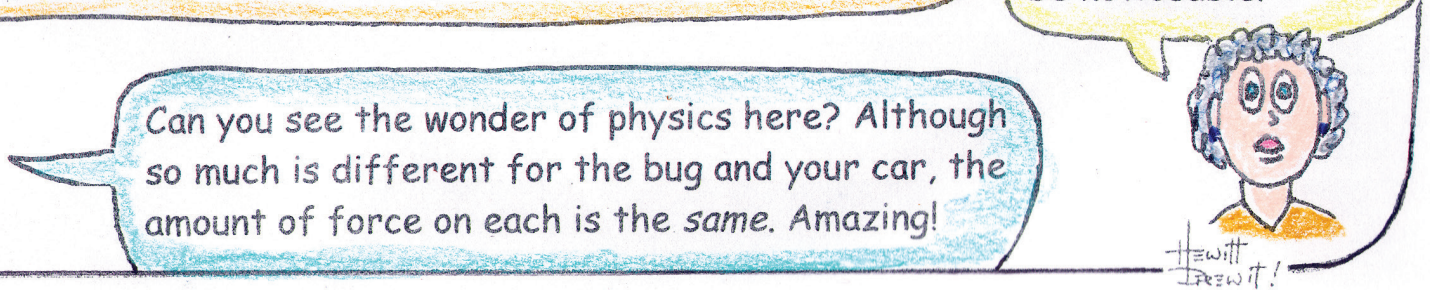

\section{Answer to December Figuring Physics}

